Setting The included studies describe a variety of sport settings and activity levels.

Participants The review comprises studies including males and/ or females of any age with ACL injury.

Assessment of Risk Factors The review comprises longitudinal studies investigating any intrinsic risk factor for future C-ACL injury.

Main Outcome Measurements C-ACL injury

Results Thirty-five moderate-to-high quality studies were eligible for meta-analysis, including up to $\sim 59000$ individuals. The following factors all independently increased the odds of sustaining a C-ACL: Returning to a high activity level (OR: 3.26, 95\% CI: 2.10-5.06), BMI < 25 (OR: 2.73, 95\% CI: 1.73-4.36), Age $\leq 18$ years (OR: 2.42, 95\% CI: 1.51-3.88), Family history of ACL injury (OR: 2.07, 95\% CI: 1.54-2.80), Primary ACL reconstruction performed $\leq 3$ months post injury (OR: 1.65, 95\% CI: 1.32-2.06), Female sex (OR: 1.35, 95\% CI: 1.14-1.61) and Concomitant meniscal injury (OR 1.21, 95\% CI: 1.03-1.42). There were no associations between the odds of sustaining a CACL injury and Smoking status, Pre-injury activity level, Playing soccer compared to other sports or Timing of return to sport.

Conclusions Demographic factors such as female sex, young age and family history of ACL injury, as well as early reconstruction and returning to a high activity level all contribute to the risk of sustaining a C-ACL injury. Studies on modifiable sensorimotor risk factors are warranted.

\section{RISK FACTORS FOR GRAFT RUPTURE AFTER ANTERIOR CRUCIATE LIGAMENT RECONSTRUCTION: A SYSTEMATIC REVIEW WITH META-ANALYSIS}

${ }^{1,2}$ Anna Cronström, ${ }^{1}$ Tengman Eva, ${ }^{1} \mathrm{~K}$ Häger Charlotte. ${ }^{1}$ Department of Community Medicine and Rehabilitation, Umeå University, Umeå, Sweden; ${ }^{2}$ Department of Health Sciences, Lund University, Lund, Sweden

\subsection{6/bjsports-2021-IOC.179}

Background Underlying factors contributing to increased risk of graft rupture after anterior cruciate ligament reconstruction (ACLR) are not well described.

Objective To systematically review intrinsic risk factors for sustaining a graft rupture.

Design A systematic review with meta-analysis according to the PRISMA guidelines. Four databases (MEDLINE, CINAHL, EMBASE, Sport Discus) were searched from inception to January 2020. Meta-analyses (random effect model) were performed and expressed as odds ratios (OR).

Setting The included studies describe a variety of sport settings and activity levels.

Participants The review comprises studies including males and/ or females of any age who have had ACLR.

Assessment of Risk Factors All longitudinal studies investigating any intrinsic risk factor for future graft rupture were included. Main Outcome Measurements Graft rupture.

Results Seventy-seven studies were eligible for meta-analysis. The following factors all independently increased the odds of sustaining a graft rupture after ACLR: Age $\leq 18$ years (OR: 3.87, 95\% CI: 2.32-6.46), higher pre-primary injury activity level (OR: 2.43, 95\% CI: 1.56-3.82), family history of ACL injury (OR: 1.98, 95\% CI: 1.50-2.62), returning to a high activity level (OR: 1.87, 95\% CI: 1.11-3.15), and increased lateral tibial slope (OR: 1.64, 95\% CI: $1.13-$ $2,38)$. None of the following factors were found to be associated with future graft rupture; sex, smoking status, generalized joint laxity, timing of surgery or return to sport (RTS), playing soccer compared to other sports, hop performance at time of RTS or concomitant meniscal or collateral ligament injuries.

Conclusions Young age, family history of ACL injury, high tibial slope and previous and current high activity level should be considered when screening for increased risk of graft rupture following ACLR. Future studies on the possible role of sensorimotor factors, e.g., muscle activation and/or strength and proprioception for future graft ruptures are warranted.

\section{PREVENTING ACL REINJURIES - IS PERSISTENT KNEE UNDERLOADING AT RETURN TO SPORTS AFTER RECONSTRUCTION THE CULPRIT?}

Argyro Kotsifaki, Vasileios Sideris, Vasileios Korakakis, Rodney Whiteley. Aspetar Orthopaedic and Sports Medicine Hospital, Doha, Qatar

\subsection{6/bjsports-2021-IOC.180}

Background Hop tests are part of the discharge criteria to determine return to sports (RTS) following anterior cruciate ligament reconstruction (ACLR). Usually, only the distance hopped is measured, but little is known regarding biomechanical performance during these tests.

Objective Describe biomechanical parameters of ACLR patients during single and triple hop for distance at the time of RTS.

Design Cross-sectional study.

Setting Laboratory, level I sport athletes.

Patients (or Participants) 28 males: 14 primary ACLR patients ( $24 \pm 6$ years) who were cleared to RTS, 14 healthy recreational athletes $(30 \pm 5)$.

Interventions (or Assessment of Risk Factors) Biomechanical differences between limbs and groups.

Main Outcome Measurements Knee power absorption and generation during the propulsion and landing phases of single and triple hops.

Results Despite similar hop distance, ACLR patients had significantly lower between-limb knee power generation $(\mathrm{d}=1.12, \mathrm{p}=0.001)$, and absorption $(\mathrm{d}=1.00, \mathrm{p}=0.002) \mathrm{dur}-$ ing single hop and less power compared to controls in both phases $(\mathrm{d}=0.78, \mathrm{p}=0.05)$. During triple hop, ACLR patients displayed less knee power generation in the first $(\mathrm{d}=1.11$, $\mathrm{p}=0.001)$ and second hop $(\mathrm{d}=1.14, \mathrm{p}=0.001)$ and less power absorption in the final landing $(d=0.73, p=0.017)$ in comparison to the uninvolved leg. Similarly, ACLR patients had less knee power generation in the first $(d=1.69$, $\mathrm{p}=0.000)$ and second hop $(\mathrm{d}=1.21, \mathrm{p}=0.004)$ and less absorption in the final landing $(\mathrm{d}=1.09, \mathrm{p}=0.008)$ compared to the control group.

Conclusions ACLR patients underload their injured knee despite meeting hop distance targets to be cleared to RTS. This pattern has not been documented previously and could explain the high risk of reinjury to the knee and other structures in the kinetic chain after RTS. 